XXIII.

Aus dem Institut für experimentelle Pathologie der deutschen Universität in Prag.

\title{
Experimentelle Untersuchungen über die Beeinflussung des Kreislaufes und der Athmung durch das Salvarsan.
}

\author{
Von \\ Priv.-Doc. Dr. Edmund Hoke und Priv.-Doc. Dr. Julius Rihl, \\ (Prag-Franzensbad) Assistenten des Institutes.
}

(Hierzu Tafel XIII und XIY.)

Einleitung.

Da die klinische Beobachtung gezeigt hat, dass bei Patienten mit kranken Circulationsorganen eine eingeleitete Salvarsantherapie nicht gleichgültig ist, ja sogar den Tod des Menschen zur Folge haben kann, erschien es wünschenswerth, die Wirkung des Salvarsans auf den Kreislituf ausführlich experimentell zu untersuchen. Schon Ehrlich (1) hat die Bedingung gestellt, „dass Leute, die ein erregbares Herznervensystem oder gar einen Klappenfehler aufweisen, ferner Leute mit Gefässdegeneration, Aneurysmen, vorübergehenden Hirnblutungen von der Behandlung auszuschliessen seien." An derselben Stelle führt Ehrlieh die bisher bekannt gewordenen Lnfälle an, die auf eine Erkrankung der Circulationsorgane bezogen werden mussten. Schliesslich war zu erwarten, dass sich durch eine genauere Analyse der Kreislaufwirkung des Salvarsans auch therapeutisehe Gesichtspunkte ergeben werden, das heisst Grundzüge für die bei derartigen Unfällen einzuschlagende Therapie. Seit der Mittheilung Ehrlich's sind noch folgende Unfälle bei Salvarsaninjectionen, die auf den Circulationsapparat Bezug haben, bekannt geworden. Schottmüller (2) berichtet über einen Todesfall nach intravenöser Injection von $0,8 \mathrm{~g}$ Ehrlich-Hata, $606 "$ in saurer Lösung. Notthafft (3) sah nach intravenöser Injection von $0,6 \mathrm{~g}$ "Ideal " eine schwere Synkope eintreten, aus der sich der Kranke nach einer halben Stunde wieder erholt hatte. Das Präparat "Ideal" steht jedoch hinsichtlich seiner Giftigkeit in der Mitte zwischen dem sauren "Hyperideal" und dem sauren Salvarsan. So erklärt es sich vielleicht, dass der Kranke mit dem Leben davon kam. Notthafft hebt nämlich hervor, dass in seinem Falle die von H. E. Hering (4) für den Mensehen aus den Thierversuchen berechnete durchschnittliche letale Dosis überschritten wurde, Hering hat aber mit dem weitaus giftigeren "Hyperideal" gearbeitet. Seit der Mittheilung 
Hering's, der vor der Verwendung der sauren IJösung warnte, wird diese wohl kaum mehr verwendet werden.

Nach H. E. Hering beträgt die Dosis letalis für das Hyperideal in saurer Lösung pro Kilo Kaninchen 0,004 bis $0,005 \mathrm{~g}$, die der alkalischen Lösung desselben Präparates ist mindestens zwanzigfach grösser. Beim Salvarsan beträgt die Dosis letalis der sauren Lösung nach unseren Versuchen $0,101 \mathrm{~g}$ (Mittelwerth aus sechs Versuchen), die der alkalischen Lösung $0,204 \mathrm{~g}$ (Mittelwerth aus fünf Versuchen) pro Kilo Kaninchen. Das Präparat "Ideal" steht in der Mitte zwischen "Hyperideal" und Salvarsan, was seine Giftigkeit für das Kaninchen anbelangt. Worauf diese auffallenden Unterschiede in der Giftigkeit dieser verschiedenen drei Präparate beruhen, ist uns nicht bekannt. Es ist aber nothwendig darauf hinzuweisen, da die verschieden angegebenen Dosen, welche zu Unglücksfällen führten, in der ersten Zeit der 606 Aera sicher zum Theil wenigstens auf den verschiedenen Grad der Giftigkeit der verwendeten Präparate bezogen werden müssen. Seit nur Salvarsan verwendet wird, kommt die Frage nach der versehiedenen Giftigkeit der einzelnen Präparate insofern nicht mehr in Betracht, als wir beim Salvarsan keine wesentlichen Unterschiede der Giftigkeit fanden. Kleine, schwächliche Thiere sind, wie zu erwarten war, empfindlicher als grosse.

\section{Methodik.}

$\mathrm{Za}$ unseren Versuchen wurden ausschliesslich Kaninchen und zwar im Gewicht von 1330 bis $3020 \mathrm{~g}$ verwendet. Immer wurde eine $1 / 2$ proc. frisch bereitete Salvarsanlösung zur Infusion benutzt. Zur Lösung des Mittels wurde 0,9 proc. Kochsalzlösung verwendet. Die zur Infusion bestimmte Lösung wurde nicht vorgewärmt, was bei der langsamen Infusion dieser relativ geringen Flüssigkeitsmengen unnöthig erschien.

Im Ganzen wurden 34 Versuche angestellt. Die Kaninchen wurden aufgespannt, die linke Carotis freigelegt und ihr centrales Ende mit einem Quecksilbermanometer verbunden, welches die Blutdruckschwankungen vermittelst eines Schwimmers auf die berusste Trommel des Hering'schen Kymographions verzeichnete. Das 'Thier war tracheotomirt und konnte mit Hilfe eines Zweiwegehahnes, der an die Trachealkanüle angesetzt war, nach dem Belieben des Experimentators einerseits in die freie Luft oder in einen Knoll'schen Athemkasten athmen, von welchem die Respirationsbewegungen auf einen M arey'schen Tambour übertragen wurden. Die Infusion erfolgte aus einer $50 \mathrm{ccm}$ fassenden graduirten Bürette durch Vermittelung einer in die rechte Vena jugularis eingebundenen Canüle.

In dem zweiten Theil unserer Versuche, welche eine genauere Analyse der im ersten Theil immer beobachteten Blutdrucksenkung zur Aufgabe hatten, wurden ausschliesslich curarisirte Thiere verwendet, um Blutdruckschwankungen, die durch Bewegung des Thieres entstehen konnten, auszuschliessen. Die Thiere wurden bis zur völligen Bewegungslosigkeit curarisirt. Zum Studium der reflectorischen Erregbarkeit der Gefässe wurde die Nasenschleimhaut, einmal der dazu freipräparirte Nervus peronaeus faradisch gereizt. Um den Effect einer Aortencompression auf den Blutdruck prüfen zu können, wurde die Aorta am Bogen ohne 
Thoraxeröffnung nach theilweiser Resection des Brustbeines soweit frei präparirt, dass sie mit einer Klemmpincette bequem gefasst werden konnte. Immer wurde die Aorta unmittelbar vor dem Abgang der Arteria subclavia sinistra comprimirt. Um den Einfluss der Splanchnicusreizung verfolgen zu können, wurde der linke Nervus splanchnicus retroperitoneal vorsichtig freigelegt, unterhalb seines Durchtrittes durch das Diaphragma durchschnitten und das periphere Ende dieses Nerven faradisch gereizt. Was die Geschwindigkeit der Infusion anbelangt, so ist diese bei den einzelnen Versuchen angegeben. $\mathrm{Zu}$ den Versuchen, welche die Analyse der Blutdrucksenkung zum Ziele hatten, wurde ausschliesslich Salvarsan in alkalischer Lösung verwendet. Die Alkalisirung geschah in der Weise, dass so viel Alkali zugesetzt wurde, bis der entstandene Niederschlag sich eben wieder löste. Dazu waren auf $120 \mathrm{ccm}$ Flüssigkeit $(0,6$ Salvarsan in $120 \mathrm{ccm} 0,9$ proc. Kochsalzlösung gelöst) immer $4,8 \mathrm{ccm}$ Normalnatronlauge nöthig.

\section{Allgemeines über das Verhalten des Blutdruckes unter dem Einfluss der verschiedenen Präparate.}

Die untersuchten Präparate verursachten sowohl in saurer wie in alkalischer Jüsung ein Sinken des Blutdruckes ${ }^{1}$ ). Der Unterschied zwischen den sauren und alkalischen Lösungen bestand wesentlich nur darin, dass die Blutdrucksenkung bei ungefähr gleich rascher Infusion bei den sauren Lösungen viel früher in Erscheinung trat und viel rascher vor sich ging als bei den alkalischen Lösungen. Nach Hyperideal in saurer Lösung kam es stets zu einem ganz rapiden Druckablall.

Wurde nach dem Eintreten einer Blutdrucksenkung die weitere Infusion sistirt, so kam es, wenn der Blutdruck nicht gar zu tief abgesunken war, zu einer Erholung desselben, allerdings niemals zur ursprünglichen Höhe.

\section{Salvarsan in alkalischer Lösung.}

Als Beispiel für das Verhalten des Blutdruckes, der Athmung und der Pulsfrequenz sei Versuch 8 angeführt.

Kaninchen $1990 \mathrm{~g}$, Salvarsan-Control-No. 87. Blutdruck im Beginne des Versuches $94 \mathrm{~mm}$ Quecksilber; Pulsfrequenz 360, Athemfrequenz 150. In etwas über einer Minute werden $12 \mathrm{ccm}$ infundirt. Druck jetzt $80 \mathrm{~mm}$ Quechsilber, Puls 330, Athemfrequenz 150. Nach der Infusion von $60 \mathrm{ccm}$ (in ca. 200 Secunden!) beträgt der Blutdruck $72 \mathrm{~mm}$, Pulsfrequenz 300, Athmung 180. In längeren Intervallen werden nun weiter 5, dann 6, dann 1, 5 und $1 \mathrm{ccm}$ infundirt. Der Blutdruck ist auf $60 \mathrm{~mm} \mathrm{ab-}$ gesunken; Puls 240, Athmung 90. Die Athmung wird weiter langsamer und tiefer, bis es schliesslich zu einer etwa 45 Secunden dauernden präterminalen Athempause kommt, welcher noch 7 terminale Athmungen mit abnehmender Tiefe folgen. Während

1) Das Auftreten einer Senkung des Blutdruckes nach Salvarsan wurde auch klinisch nachgewiesen, zuerst von $\mathrm{Nicolai}$ nach subcutaner Application, später von Gennerich, Zur Technik und Contraindication der Salvarsanbehandlung, Münchener med. Wochenschr. No, 52, 1910 und Sieskind, Das Verhalten des Blutdruckes bei intravenösen Salvarsaninjectionen, Münchener med. Wochenschr. No. 11, 1911, nach intravenöser Application. 
dieser präterminalen Athempause sank der Blutdruck weiter von $58 \mathrm{~mm}$ Quecksilber auf $12 \mathrm{~mm}$ ab unter zunehmender Verlangsamung der Herzaction. Nach etwa zwei Minuten hatte die Druckcurve die Abscisse erreicht, wïhrend das Herz noch schwach weiter schlug, wie die sofort vorgenommene Eröffnung des Thorax zeigte (Fig. I).

\section{Salvarsan in saurer Lösung.}

Versuch 7. Kaninehen $2000 \mathrm{~g}$ sohwer; Salvarsan "63“.

Blutdruck im Beginne des Versuches $106 \mathrm{~mm}$ Queckisilber, Pulsfrequenz 360, Atbemfrequenz 90 . Es wurden nun in Intervallen von 9 bis über 40 Secunden langsam $0,5,0,5,4,5,3,8 \mathrm{~cm}$ der sauren Salvarsanlösung infundirt. Blutdruck jetzt $112 \mathrm{~mm}$, also höher als im Beginne der Infusion, Puls 300, Athemfręuenz 90. Nach weiteren 100 Secunden werden $\bar{I} \mathrm{ccm}$ infundirt. Daranf sinkt der Druck allmählich auf $88 \mathrm{~mm} \mathrm{ab}$, Puls 300, Athemfrequenz 240. Nach Einlauf von 1 ccm beträgt der Druck $50 \mathrm{~mm}$. Nun tritt zunehmende Athemverlangsamung auf, es kommt zur präterminalen Athempause und dann za den terminalen Athemzügen. Bei einem Druck von $26 \mathrm{~mm}$ Quecksilber kommt es zu dauerndem Athemstillstand.

\section{III. „Hyperideal" in saurer Lösung.}

Versuch 16. Kaninchen $2000 \mathrm{~g}$ schwer; Präparat lfyperideal $0,3 \mathrm{ccm}$ in $60 \mathrm{ccm}$ 0,9 proc. Kochsalzlösung gelöst.

Blutdruck im Beginne des Versuches $102 \mathrm{~mm}$ Quecksilber, Pulsfrequenz 300; Athmung 150. In 12 Secunden wurden $2,5 \mathrm{ccm}$ infundirt. Der Druck stürzt in wenigen Secunden auf $28 \mathrm{~mm} \mathrm{ab}$, die Herzthätigkeit wird unregelmässig, Athmung 150 . Präterminale Athmungen treten auf und noch vor dem Absinken der Blutdruckcurve zur Abscisse kommt es zum dauernden Athemstillstand (Fig. II).

Das Hyperideal in alkalischer hösung steht dem Salvarsan viel näher, nur ist es, wie in der línleitung erwähnt wurde, gjftiger.

\section{Analyse der Blutdrucksenkung bei Salvarsan in alkalischer Lösung.}

Das Absinken des Carotisdruckes unter dem Einflusse des Salvarsans konnte durch verschiedene Möglichkeiten bedingt sein. Das Gift konnte entweder primär das Herz schädigen, oder es konnte die Gefässe beeinflussen oder war es möglich, dass beide eben genannten Wirkungsweisen sich kombinirten. Zur Differenzirung wurden nun folgende Versuche angestellt und zwar ausschliesslich am curarisirten Thiere $\left.{ }^{1}\right)$. 1. Die Aortencompression. Bewirkte dieser lingriff bei bereits eingetretener erheblicher Senkung des Blutdruckes noch eine deutliche Stcigerung desselben, so konnte im Herzen die Ursache der Drucksenkung nicht gelegen sein. 2. Die Prüfung der reflectorischen Erregbarkeit der Gefässe durch faradische Reizung der Nasenschleimhaut des Thieres (Trigeminus) oder durch faradische Reizung des centralen Stumpfes des zu diesem Zwecke freigelegten Nervus ischiadicus, ferner die Prüfung der centralen Erregbarkeit des Vasoconstrictorencentrums durch Dyspnoe. Zeigte sich ein Versagen der Wirkung der reflectorischen und centralen Reizung der Gefässe, so konnte die Ursache dafür central oder peripher bedingt sein. Um dies zu entscheiden, wurden 3 . Versuche angestellt, die den Erfolg

1) Bei den curarisirten Thieren war die tödtliche Dosis des Salvarsans weitaus geringer als sie in der Einleitung angegeben ist. 
einer peripheren Reizung des Nervus splanchnicus studiren sollten. Zeigte sich ein Versagen der letzteren, so konnte dies nur durch ein peripheres Angreifen des Giftes erklärt werden.

\section{Aortencompression nach Trigeminusreizung.}

Versuch 29. Kaninchen $1860 \mathrm{~g}$. Salvarsan "100“.

Blutdruck im Beginne des Versuches $80 \mathrm{~mm}$ Quecksilber, Pulsfrequenz 252. Das Aussetzen der kïnstlichen Respiration führt zu einer Blutdrucksteigerung bis auf $126 \mathrm{~mm}$ Quecksilber, die Abklermmung der Aorta zu einer solchen von $140 \mathrm{~mm}$. Nun wurde die Nasenschleimhaut des Thicres faradisch gereizt bei einem Rollenabstand von $8 \mathrm{~cm}$; es steigt der Druck von 80 auf $112 \mathrm{~mm}$. Um 11 Uhr 12 Minuten wurde mit der Salvarsaninfusion begonnen. Als erster Fifect der Infusion zeigt sich das Kleinerwerden und schliessliche Verschwinden von Blutdruckschwankungen, die aller Wahrscheinlichkeit nach als Traube-llering'sche Wellen anzusprechen sind. Um 11 Uhr 21 Min. waren $38 \mathrm{ccm}$ Salvarsan infundirt worden. Der Blutdruck beträgt jetzt $70 \mathrm{~mm}$ Quecksilber, Pulsfrequenz 192. Bei einem weiteren Absinken des Druckes auf $45 \mathrm{~mm}$ Wuecksilber versayt die Reizung der Nasenschleimhaut bei einem Rollenabstand von $8 \mathrm{~cm}$ schon fast vollständig. Um 11 Uhr $27 \mathrm{Min}$. werden in Intervallen von 30 Sec. je 1,5, dann 1, 1, $1 \mathrm{ccm}$ Salvarsan, um 11 Uhr 32 Min. $2 \mathrm{ccm}$ und nach 35 Sec. noch $4 \mathrm{ccm}$ Salvarsan infundirt. Trotzdem kommt es zu einer, allerdings nur vorübergehenden lirholung des Thieres, indem sich der Blutdruck wieder auf $70 \mathrm{~mm}$ (uecksilber erhebt; auch die Reizung der Nasenschleimhant bei dem früher gewählten Rollenabstand wird wieder wirksam und fübrt zu einer Druckerhebung auf $82 \mathrm{~mm}$. Um 11 Uhr 35 Min. sinkt der Blutdruck nun continuirlich ab. Bei einem Blutdruck von $25 \mathrm{~mm}$ versagt schliessich die Reizung der Nasenschleimhaut auch bei 0 Rollenabstand. 'Trotzdem erweist sich die Aortencompression noch als wirksam, die noch zu einer Druckerhebung auf $58 \mathrm{~mm}$ führt; ja selbst in Ultimis bei einem Druck von nur mehr $14 \mathrm{~mm}$ lionsut es nach der Aortencompression noch zu einer Druckerhebung auf $40 \mathrm{~mm}$ (Fig. III bis incl. VI).

Der Versuch zeigt in eindeutiger Weise, dass eine primäre Herzschädigung sicher nicht die llauptursache der Blutdrucksenkung sein $\operatorname{kann}^{1}$ ); sie ist vielmehr in einer Beeinträchtigung des Gefässtonus zu suchen, worauf das allmähliche Versagen der Wirkung der reflectorischen Vasoconstrictorenreizung hinweist.

\section{Faradische Reizung des peripheren Endes des Nervus splanchnicus und Einfluss der dyspnoischen Vasoconstrictorenreizung.}

Da das Versagen der Wirkung der reflectorischen Vasoconstrictorenreizung, wie schon erwähnt, central oder peripher bedingt sein konnte, wurden zwei Versuche mit peripherer Splanchnicusreizung vorgenommen.

In diesen Versuchen wurdo in Parallele mit der peripheren Splanchnicusreizung auch immer das Verhalten der dyspnoischen Vasoconstrictorenroizung geprüft.

Versuch 33. Kaninchen $3380 \mathrm{~g}$ schwer, Salvarsan „100“. Beginn der Untersuchung um 4 Uhr 48 Min.

1) Die in unseren Versuchen festgestelite geringe Schädigung des Herzens ist um so bemerkenswerther, als bei denselben das Gift in ein dem Herzen so naheliegendes Gefäss infundirt wurde, also mehr unverdünnt in das Herz gelangte. 
Blutdruck 95, Puls 390. Bei $15 \mathrm{~cm}$ Rollenabstand und 5 Sec. langer Splanchnicusreizung kommt es zu einer Drucksteigerung bis auf $130 \mathrm{~mm}$ Quecksilber. Das Aussetzen der künstlichen Ventilation durch $20 \mathrm{Sec}$. führt zu einer Drucksteigerung bis auf $170 \mathrm{~mm}$ Quecksilber. Einige Secunden nach $5 \mathrm{Uhr}$ wurde mit der Infusion der Salvarsanlösung begonnen, in der Weise, dass in ca. 2 Minuten immer $10 \mathrm{ccm}$ einliefen. Bis 5 Uhr $7 \mathrm{Min}$. waren $25 \mathrm{ccm}$ eingeflossen. Die Infusion wurde nun sistirt. Druck nicht ganz $100 \mathrm{~mm}$ Quecksilber, Pulsfrequenz 480. Um 5 Uhr 11 Min. war der Druck auf $90 \mathrm{~mm}$ abgesunken; Puls 450 . Die durch 5 Sec. noch bis $15 \mathrm{~cm}$ Rollenabstand ausgeführte Splanchnicusreizung steigert den Druck auf $106 \mathrm{~mm}$. Um 5 Uhr $19 \mathrm{Min}$. wird wieder durch $20 \mathrm{Sec}$. die künstliche Athmung ausgesetzt, was eine Drucksteigerung bis auf $186 \mathrm{~mm}$ Quecksilber zur Folge hat. Um 5 Uhr $24 \mathrm{Min}$. wird neuerdings Salvarsan infundirt so zwar, dass bis 5 lihr 27 Min. $40 \mathrm{ccm}$ eingelaufen waren. Die Infusion wird wieder unterbrochen. Druck $86 \mathrm{~mm}$ Quecksilber, Puls 450 . Um 5 Uhr 30 Min. wird abermals der Splanchnicus gereizt. Bei $15 \mathrm{~cm}$ Rollenabstand nach 5 Sec. langer Reizung kann eine Druckerhebung bis anf $100 \mathrm{~mm}$ Quecksilber beobachtet werden. Das Aussetzen der künstlichen Respiration (durch 20 Sec.) führt zu einer Drucksteigerung von $96 \mathrm{~mm}$. Um 5 Uhr 33 Min. wird neuerlich Salvarsan infundirt. Druck $96 \mathrm{~mm}$, Puls 450 . Um 5 Uhr $36 \mathrm{Min}$. waren im Ganzen $55 \mathrm{ccm}$ der Lösung eingeflossen. Das Aussetzen der künstlichen Ventilation durch 20 Sec. führt zu einer Druckerhebung von vorher $72 \mathrm{~mm}$ auf $97 \mathrm{~mm}$. Die Splanchnicusreizung steigert den Druck von $60 \mathrm{~mm}$ auf 86 . Nun sinkt der Druck rasch ab; bei $25 \mathrm{~mm}$ Druck versagt das $\Lambda$ ussetzen der künstlichen Ventilation schon vollständig, während die Splanchnicusreizung $(15 \mathrm{~cm}$ Rollenabstand, Reizungsdauer 5 Sec.) den Druck immerhin noch von 20 anf $25 \mathrm{~mm}$ Quecksilber erhebt. Auch bei einem Druck von nur mehr $12 \mathrm{~mm}$ führt die Splanchnicusreizung bei Rollenabstand 0 noch zu einer allerdings sehr geringfügigen aber immerhin deutlichen Drucksteigerung (Fjg. VIL-XI).

Aus diesen Versuchen geht hervor, dass 1. die periphere Erregbarkeit des Splanchnicus unter dem Einfluss des Salvarsans allmählich abnimmt; 2. der Splanchnicus noch bei vollständig paralytisehem Drucke erregbar ist, während die Erregbarkeit des Vasoconstrictorencentrums durch Dyspnoe schon bei einem höheren Drucke erlischt. ${ }^{1}$ )

\section{Schlussfolgerungen.}

Die Wirkungsweise des Salvarsans in alkalischer Lösung auf die Kreislauforgane ist eine complexe. Die Hauptcomponente ist wohl eine Beeinflussung der nervösen Centralorgane, wie aus dem Abnehmen des Effektes der dyspnoischen Vasoconstrictorenreizung zu einer Zeit, wo sich die periphere Splanchnicusreizung noch als wirksam erweist, hervorgeht. Für eine Beeinflussung des centralen Nervensystems spricht ferner das Verschwinden der Traube-Hering'schen Wellen aus der Blutdruckcurve, auch ferner der Umstand, dass es mitunter schon bei relativ hohem Drucke zum Athemstillstand kan. Der zweite Factor der Salvarsanwirkung ist ein peripherer, wie das allmählige Versagen der peripheren Erregbarkeit des Nervus splanchnicus mit Sicherheit beweist. Als

1) Die Erregbarkeit des peripheren Vagus wurde durch das Salvarsan nicht beeinträchtigt. Wir konnten uns durch unmittelbare Inspection des nach Absinken des Blutdruckes auf die Abscisse freigelegten Herzens überzeugen, dass periphere Vagusreizung noch eine deutliche Verlangsamung der Vorhoffrequenz bedingte. 
dritte und wohl im Vergleich mit den eben genannten am wenigsten bedeutungsvolle Componente ist dann die direct herzschädigende Wirkung des Salvarsans in Betracht zu ziehen.

Wir gelangen so bezüglich der Kreislaufwirkung des Salvarsans zu ganz ähnlichen Ergebnissen wie Pistorius (5) bezüglich der Kreislaufwirkung der arsenigen Säure.

Pistorius, der unter Böhm's Leitung die von Böhm und Unterberger im Jahre 1874 veröffentlichten Untersuchungen über die arsenige Säure überprüfte und vervollständigte, sagt: "Die Beobachtung, dass im ersten Anfang die Wirkung der Athemsuspension, dann die der Reizung des centralen Ischiadicusstampfes, endlich die der directen Rückenmarksreizung in Wegfall kornmen, legt uns die Annahme allmähliger Lähmung der Organe der Medulla oblongata sehr nahe. Die Splanchnicusversuche anderseits weisen wohl ebenso zwingend auf eine Veränderung in der Contractilität der Unterleibsgefässe hin. Berücksichtigen wir ferner noch, dass ohne Zweifel auch die Herzthätigkeit durch unser Gift beeinflusst wird, so stehen wir vor einem so sehr verwickelten Problem."

Wir kommen daher zu dem Schlusse, dass die Giftwirkung des Salvarsans wesentlich eine Arsenwirkung ist.

Es wirft sich nunmehr die Frage auf, wie weit die hier mitgetheilten Resultate der Analyse der Blutdrucksenkung in therapeutischer Hinsicht zu verwerthen wären.

Wie die centralen Componente an erster, die periphere nach unseren Versuchen an zweiter Stelle steht, so kommen vielleicht in erster Linie Champher und Strychnin, dann das gefässverengernde Adrenalin in Betracht.

\section{Literatur.}

1) Ehrlich, Die Salvarsantherapie. Rückblicke und Ausblicke. Münchener med. Wochenschr. 1911. Bd. 58. S. 1.

2) Schottmüller, Ebendas. 1910. Bd. 57. S. 2724.

3) Notthafft, Zur Frage der Gefahr endovenöser Einspritzungen saurer Lösungen von Salvarsan etc. Deutsche med. Wochenschr. 1911. Nr. 5. S. 202.

4) Hering, H. E., Experimentelle Erfahrungen über die letale Dosis der sauren Lösung von Ehrlich-Hata 606. Münchener med. Wochenschr. 1910. Bd.57. Nr.50.

5) Pistorius, Beiträge zur Pathologie der acuten Arsenvergiftung. Arch. f. experim. Pathol. u. Pharmakol. 1883. Bd. 16. S. 188.

\section{Erklärung der Figuren auf Tafel XIII und XIV.}

$\mathrm{R}=$ Athmung.

$\mathrm{C}=$ Carotisdruck (Hg Manometer).

Auf der untersten Geraden ist die Zeit in Secunden, auf der zweiten und dritten Geraden von unten sind die während des Versuches ausgeführten Eingriffe markirt.

Figur I (Versuch 8). Infusion von 0,5 proc. alkalischer Salvarsanlösung, Auftreten der praeterminalon Athempause bei relativ hohem Blutdruck; Absinken des Blutdruckes während der praeterminalen Athempause und des Stadiums der terminalen Athmung. Figur IB schliesst sich unmittelbar an IA an. 
Experim. Untersuchungen üb. d. Beeinflussung d. Kreislaufes durch d. Salvarsan. 339

Figur II (Versuch 16). Infusion von 0,5 proc. saurer Hyperideallösung. Nach Infusion von $2,5 \mathrm{ccm}$ kommt es zum rapiden Druckabfall, zu einer Verlangsamung der Athmung, zur praeterminalen Athempause und zu den terminalen Athmungen. Zwischen dem Ende von II A und dem Beginne von IIB sind 43 Secunden verstrichen, während welcher keine Athmung erfolgte.

Fig ur III bi in ol. VI (Versuch 29). Figur IIl Blutdrucksteigerung nach Aortencompression (vor der Infusion).

Figur IV. Blutdrucksteigerung nach faradisoher Reizung der Nasenschleimbaut bei Rollenabstand 8 (vor der Infusion).

Figur V. Nahezu vollständiges Versagen der Reizung der Nasenschleimhaut bei Rollenabstand 8 nach Infusion von $38 \mathrm{ccm}$ alkalischer 0,5 proc. Salvarsanlösung (innerhalb 9 Minuten).

Figur VI. Vollständiges Versagen der Nasenschleimbautreizung bei Rollenabstand 0; erhebliche Blutdrucksteigerung nach Aortenabklemmung. Figur VI wurde 31 Minuten nach Figur $V$ aufgenommen; in der Zwischenzeit waren noch weitere $8,5 \mathrm{ccm}$ der Salvarsanlösung infundirt worden.

Figur VII bis XI (Versuch 33).

Figur VII. Reizung des peripheren Splanchnicusstumpfes bei Rollenabstand 15 (vor der Infusion).

Figur VIII. Aussetzen der künstlichen Ventilation vor der Infusion.

Figur IX. Aussetzen der künstlichen Athmung; periphere Splanchnicusreizung bei Rollenabstand 15. Figur IX wurde 48 Minuten nach Figur VIIl aufgenommen, in der $Z$ wischenzeit waren $55 \mathrm{ccm}$ Salvarsan $(0,5 \mathrm{pCt}$. in alkalischer Lösung) infundirt worden.

Figur X. Aussetzen der künstlichen Ventilation hat keinen Effekt mehr, während die Splanchnicusreizung bei einem Rollenabstand von $15 \mathrm{~cm}$ noch eine deutliche Drucksteigerung hervorruft. Figur $\mathrm{X}$ wurde etwa 1,5 Minuten nach Figur IX aufgenommen, obne dass inzwischen neuerlich Salvarsan infundirt worden wäre.

Figur XI. Splanchnicusreizung bei Rollenabstand 0 bewirkt eine zwar geringe, aber immerhin deutliche Steigerung des bis auf $12 \mathrm{~mm}$ abgesunkenen Druckes. 


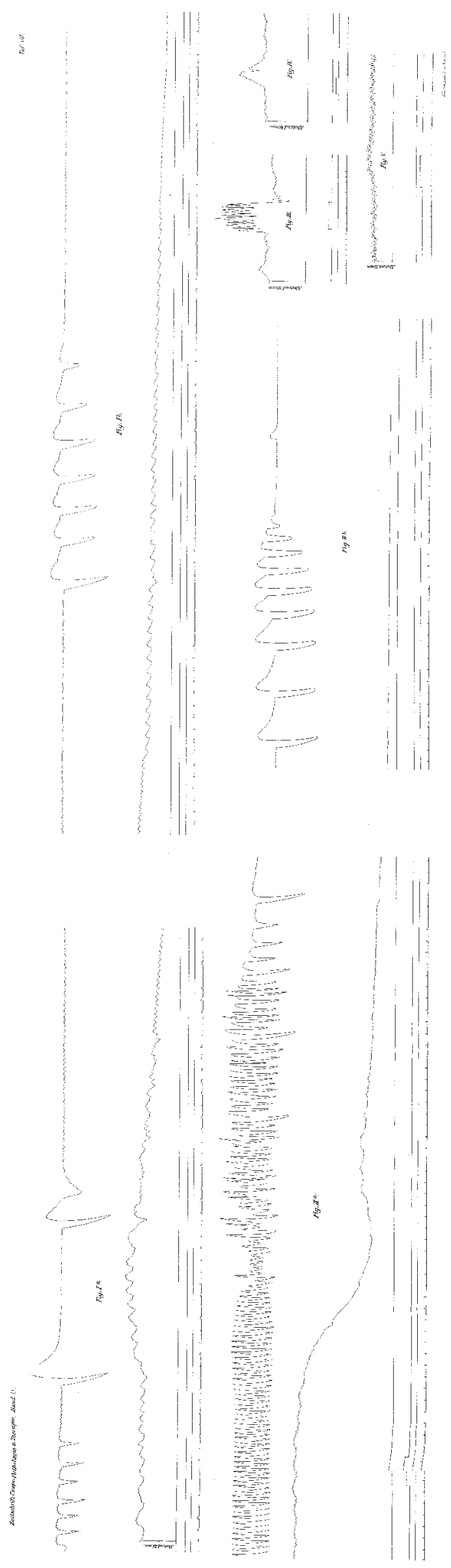




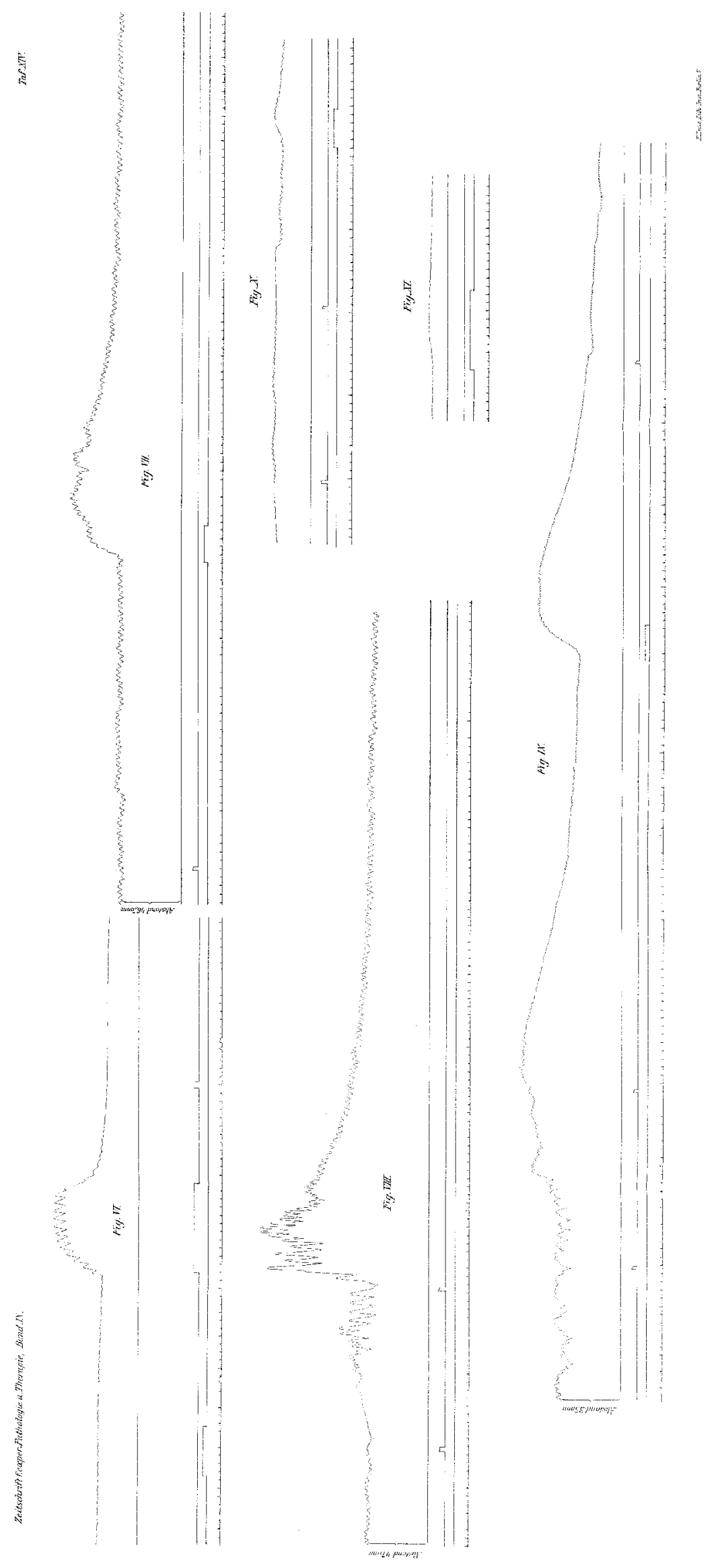

